\title{
The Calibration Units of the KM3NeT neutrino telescope
}

\section{Van Elewyck ${ }^{*}, a$, P. Keller ${ }^{b}$ and M. Lindsey Clark $^{a}$ for the KM3NeT Collaboration}

a APC, Université Paris Diderot, CNRS/IN2P3, CEA/Irfu, Observatoire de Paris, Sorbonne Paris

Cité, France

${ }^{b}$ CPPM, Aix-Marseille Université, CNRS/IN2P3, Marseille, France

E-mail: elewyck@apc.univ-paris7.fr, keller@cppm.in2p3.fr,

lindseyceapc.in2p3.fr

\begin{abstract}
$\mathrm{KM} 3 \mathrm{NeT}$ is a network of deep-sea neutrino telescopes to be deployed in the Mediterranean Sea that will perform neutrino astronomy and oscillation studies. It consists of three-dimensional arrays of thousands of optical modules that detect the Cherenkov light induced by charged particles resulting from the interaction of a neutrino with the surrounding medium.

The performance of the neutrino telescope relies on the precise timing and positioning calibration of the detector elements. Other environmental conditions which may affect light and sound transmission, such as water temperature and salinity, must also be continuously monitored. For these purposes, KM3NeT foresees the deployment of several dedicated Calibration Units, a few of which equipped with a semi-autonomous and recoverable inductive line supporting the environmental monitoring instruments. This contribution describes the technical design of the first Calibration Unit, to be deployed on the French site as part of KM3NeT Phase 1, as well as the purpose and characteristics of the different instruments that it will support.
\end{abstract}

The 34th International Cosmic Ray Conference,

30 July- 6 August, 2015

The Hague, The Netherlands

\footnotetext{
* Speaker.
} 


\section{Introduction}

The KM3NeT Collaboration is currently constructing the first phase of a next-generation neutrino telescope on two sites in the Mediterranean Sea: KM3NeT-Fr near Toulon, France, and KM3NeT-It near Capo Passero in Sicily [1]. Each site will host a three-dimensional array of thousands of photosensors that will detect the Cherenkov light resulting from neutrino interactions in the vicinity of the detector. The French site will be mainly dedicated to the study of oscillation effects with $\sim \mathrm{GeV}$ atmospheric neutrinos (ORCA) while the Italian one will focus on high-energy ( $\mathrm{TeV}-\mathrm{PeV}$ ) neutrino astronomy (ARCA).

The KM3NeT detector relies on a novel design for its Digital Optical Modules (DOMs), housing 31 three-inch photomultiplier tubes enclosed in a glass sphere [2]. The DOMs are distributed along vertical flexible strings anchored to the seabed. The anchor (or base) at the bottom of the string is the interface with the seabed infrastructure: it supports the interlink cable, equipped with a wet-mateable connector, and the base container, which houses a power converter and dedicated optical components. Each string with its anchor constitutes one detection unit (DU) of the KM3NeT detector. For storage and deployment, the string supporting the DOMs is coiled around a large spherical frame called launcher vehicle, fixed to the string anchor [3]. A surface boat is used to deploy the furled launcher vehicles at their designated position on the seabed. A remotely operated vehicle (ROV) deploys and connects the interlink cables from the base of a DU to one of the junction boxes (or another DU), that provide connections for power and data communication.

The performance of a neutrino telescope crucially depend on the accurate time and energy calibration of the detector elements, as well as on the precise knowledge of their position, which is affected by sea currents. Based on the ANTARES experience, the required angular accuracy on the event reconstruction can only be achieved with a sub-ns timing accuracy, which translates into a positioning precision of about $10 \mathrm{~cm}$ for the DOMs. Measurements of water properties are also important for evaluating the detection efficiency. The water transparency affects the propagation of Cherenkov light in sea water, reducing the visible volume and worsening the angular resolution. In-situ calibration procedures must therefore be defined in order to monitor the evolution of these parameters with time and environmental conditions (temperature, sea currents, bioluminescence...). This will be achieved using monitoring tools and probes installed on the detector elements (DOMs and anchors) and on dedicated Calibration Units (CUs) distributed among the detector lines.

The KM3NeT positioning system is based on a network of acoustic transmitters (beacons) and receivers (hydrophones) that will be distributed in the detector installation field, on the CUs (emitter an receiver), DU bases (receiver) and standalone tripods, forming a long-baseline reference system for the determination of the DOM positions by triangulation [4]. The timing calibration will be performed thanks to LED beacons mounted on the DOMs, and to a few laser beacons installed on the CUs [5]. This beacon network will be also used to monitor the water transparency. The other external parameters (sea currents, temperature, salinity...) will be continuously monitored by commercial probes installed on the CUs. Sea currents, which induce displacements of the detection lines, will be measured by commercial Acoustic Doppler Current Profilers (ADCP), in order to establish possible correlations with other environmental parameters such as the optical bioluminescent activity. Such correlations are also of great interest for the Earth and Sea Sciences, as already illustrated by joint publications using ANTARES data [6]. 
Section 2 describes the general design of the first KM3NeT CU, to be deployed on the KM3NeTFr site along with the first lines of the ORCA detector in 2016. The two main components of the $\mathrm{CU}$, namely the Calibration Base and the Instrumentation Unit, are presented in Sections 3 and 4 respectively. Perspectives on the deployment and operation of the KM3NeT CUs are briefly discussed in Section 5.

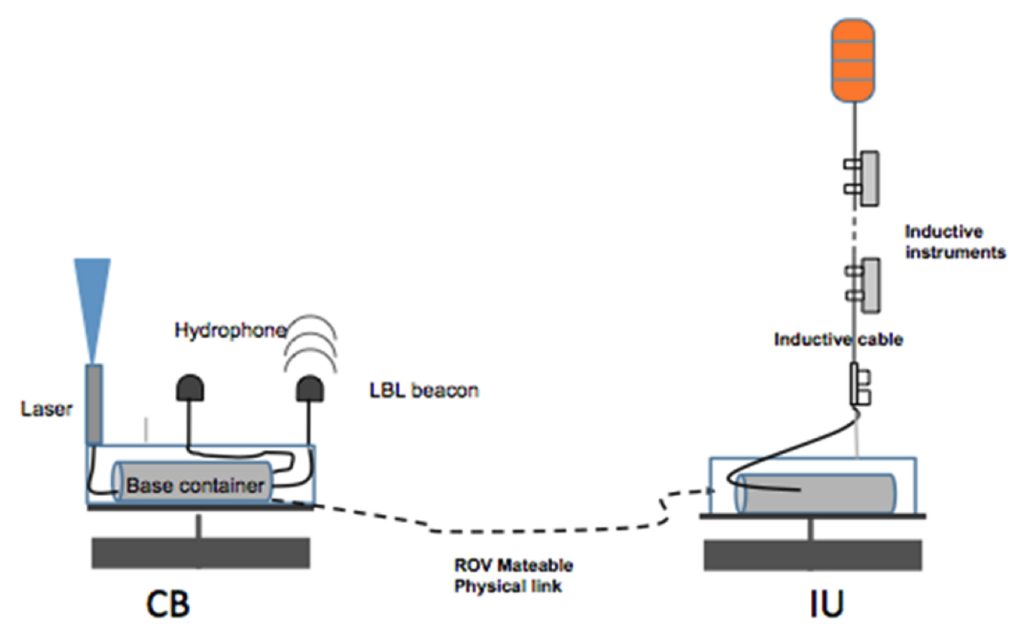

Figure 1: General design of the KM3NeT Calibration Unit.

\section{General description of the Calibration Unit}

The general design of a KM3NeT Calibration Unit is shown in Figure 1. It will comprise two sub-systems: a Calibration Base (CB) and an Instrumentation Unit (IU), powered through the $\mathrm{CB}$. The $\mathrm{CB}$ will host the detector calibration devices, namely a laser beacon, a long-baseline acoustic beacon and a hydrophone. The IU consists in an instrumentation base (IB) incorporating an electronics interface board and an inductive modem to be connected to an inductive, semiautonomous and recoverable instrumentation line (IL) equipped with environmental monitoring instruments distributed in clusters at different depths. The height of the IL will be adapted to the detector configuration (750 $\mathrm{m}$ for ARCA and 200-250 $\mathrm{m}$ for ORCA are currently being considered). The CB and the IB will be separated by approximately $80 \mathrm{~m}$. The default option for the CB-IU connection is to use a ROV mateable electrical Ethernet connector, but the possibility to use an acoustic modem is also under study, as discussed in Section 4.2.

The full CUs equipped with a calibration base and an instrumentation unit will be placed in peripherical positions around the detector, in order to allow easy periodical recovery and redeployment of the IU. In a sparse detector configuration (ARCA), CBs alone can also be distributed more homogeneously inside the detector, thereby ensuring a complete coverage of the whole network of strings. The possibility to attach standard detection lines to the bare CBs is also being considered and taken into account in the $\mathrm{CB}$ mechanical design. The first KM3NeT CU to be deployed on the French site will be a full unit with a $\mathrm{CB}$ and an $\mathrm{IU}^{1}$.

${ }^{1}$ The Phase 1 of the KM3NeT-It site foresees to deploy the laser and acoustic beacons directly on the first junction box, but the infrastructure will be harmonized to the standard $\mathrm{CU}$ for the subsequent phases of the project. 


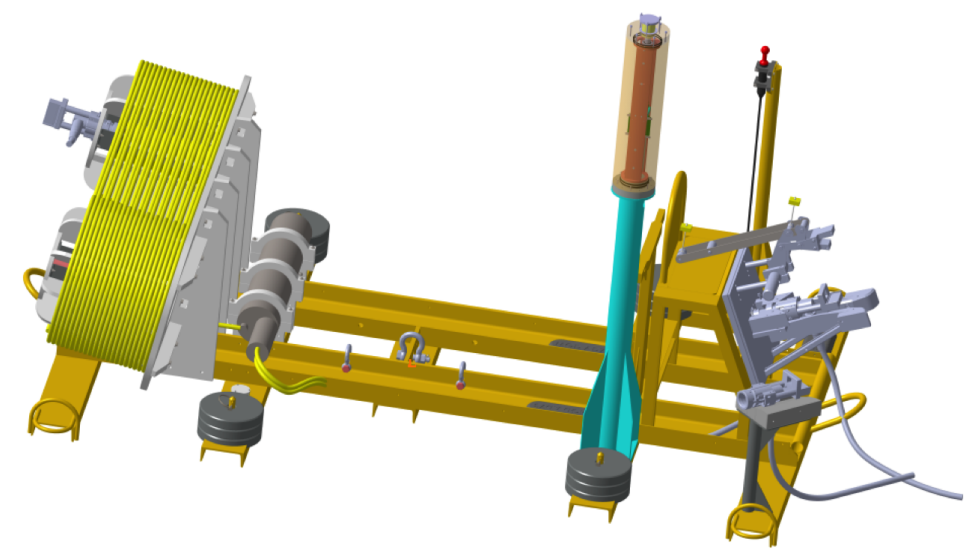

Figure 2: Preliminary design of the Calibration Base, showing the mechanical frame (dark yellow), the interlink cable used to connect the CU to a junction box or a standard DU (yellow), the cylindrical base container (dark grey), the hydrophone (red) and the mast (blue) supporting the Laser Beacon (orange).

\section{The Calibration Base}

The CB will consist of a DU base modified so as to host the following instruments on its external frame:

- a Laser Beacon used for time calibration and measurement of water optical properties

- an acoustic receiver (hydrophone) used to provide the position of the CB on the seafloor

- an acoustic long-baseline emitter that serves for the DOM positioning

- possibly an acoustic modem for connection to the IU, if this solution is retained

A preliminary design for the mechanical frame of the CB is presented in Figure 2, showing the additional mast that will support the laser beacon. This mast has been designed to withstand the laser weight (approx. 50kg) and positioned on the structure so that the center of gravity could be adjusted using the four piles of weights. All the electronics, optical and power devices are enclosed in a Titanium cylindrical Base Container attached to the CB frame. This container will in particular incorporate

- one KM3NeT Central Logic Board (CLB) [7] and its FPGA Mezzanine Card (FMC), for data acquisition and transmission

- two optical amplifiers (EDFA) to read and write from/to the optical fibers

- a power conversion board to power all the CB elements and the IU

All devices except for the hydrophone will be connected to the CLB through RS232 and RS485 connectors embedded on the FMC auxiliary board. In case the communication with the IU is through an Ethernet cable, an additional Ethernet/RS485 convertor will be needed. In addition, a photodiode will be encapsulated near the base of the Laser Beacon and connected to the CLB in order to timestamp the emitted flash of light. A preliminary synoptic view of the electronics component and their connections to the various CB instruments is shown in Figure 3.

\subsection{The acoustic devices}

Each CB will have a Long-Baseline acoustic beacon that has its own modulation signature 


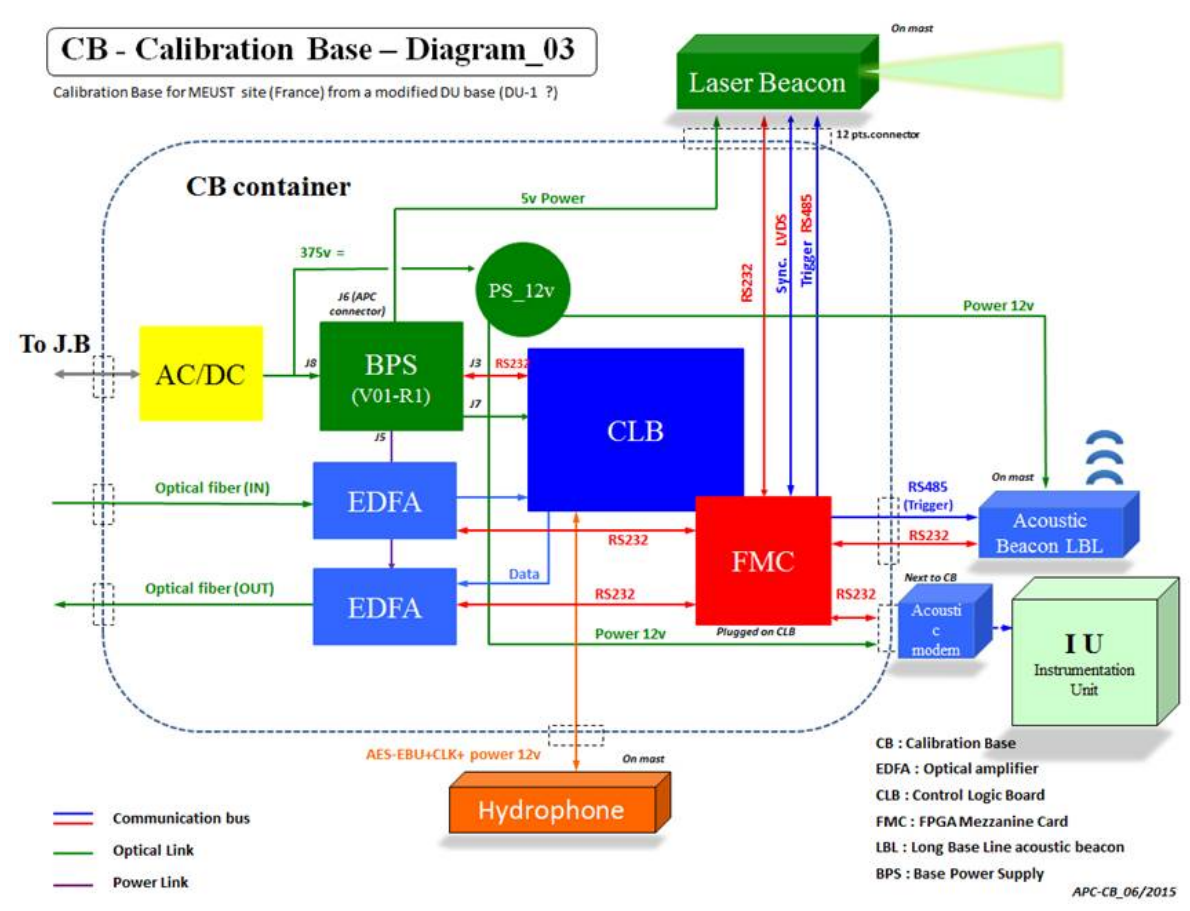

Figure 3: Preliminary electronics scheme for the Calibration Base, with the acoustic option for the communication with the IU. The hydrophone, the acoustic and laser beacons and (in this configuration) the acoustic modem are external to the Base Container.

carried by a 10 to $40 \mathrm{kHz}$ signal, depending on the range needed to allow for the simultaneous detection by one DOM hydrophone of several signals in order to determine its position by triangulation. The mechanical frame of the device is divided in two parts: one supporting the acoustic transducer and another part consisting of an Aluminum shielded container designed to host the electronics board of the device and to resist pressures up to $400 \mathrm{~atm}$. The long-baseline acoustic beacon will be connected by means of a cable with MC-IL-6F/MC-IL-6M connectors to the CB container and then linked to the CLB via an RS232 connection. Further detail on the elements of the acoustic positioning system of KM3NeT are provided in [4].

\subsection{The laser beacon}

The laser chosen for the calibration of KM3NeT DOMs is the Nd-YAG model STG-03E-040 and its associated control board MLC-03A-BP1 from Teem Photonics [8]. It emits at a wavelength of $532 \mathrm{~nm}$ (green) and provides high peak power $(>6 \mathrm{~kW})$ pulses of duration $<0.5 \mathrm{~ns}$; it can be triggered at a maximum repetition rate of $4 \mathrm{kHz}$.

During the experiment, the Laser can be in 3 different states: off (no current is required), idle, i.e. ready to emit light (the current requirement is then $\sim 0.3 \mathrm{~A}$ at $12 \mathrm{~V}$ ), or firing (the mean operation current being $630 \mathrm{~mA}$ and the peak current $\sim 2.1 \mathrm{~A}$, both at $12 \mathrm{~V}$ ). This current cannot be drawn directly from the detector power supply, so that a local power accumulator is needed. Nickel-metal hydride rechargeable batteries have been chosen in view of their optimal capacity/volume coefficient and high number of charging cycles $(\sim 1800)$. The battery pack will 


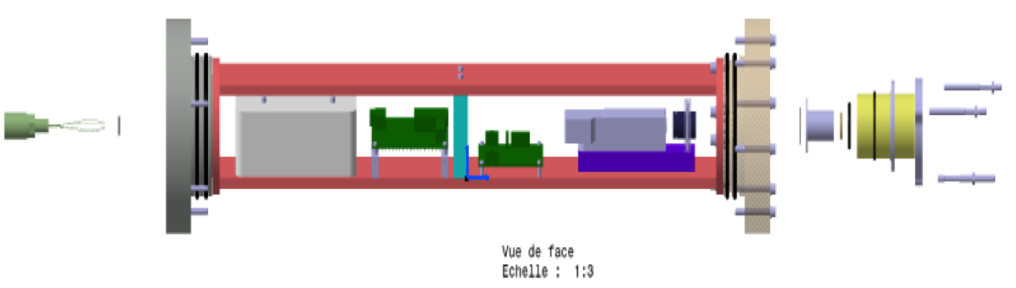

Figure 4: Exploded view of the Laser Beacon container, from left to right: connector (light green), battery pack (grey), electronics cards (dark green), laser (grey) and its support (purple), attenuator (black), opal diffuser (grey) and crystal rod (yellow).

be under charge during the "off" state of the Laser beacon (which can last for several days) with a constant charge current of $100 \mathrm{~mA}$. A custom electronics card, the Laser Power Management and Interface (LPMI), communicates with the FMC card via an RS232 connection, allowing to define the status of the Laser and the charge control.

In order to avoid the saturation of PMTs in DOMs close to the Laser Beacon, the output optical power of the Laser can be adjusted by means of an attenuator placed between the Laser and the optical window. This attenuator is an optical liquid crystal device in which the polarizing axis can be controlled by means of an external electrical signal from the LPMI. When the polarizing axis is orthogonal to the Laser light polarisation, the attenuation is maximal. The chosen attenuator is the model LVA-100-532 of Photon lines [9]. An OPAL diffuser (Newport 10DIFF-VIS [10]) is placed between the attenuator and the optical window to ensure uniformity in the angular distribution of the emitted light.

The laser beacon will be mounted in an Aluminium (class 6) cylindrical container with protective coating, as shown in Figure 4. One of the sides of the container hosts the connector, while the other one supports the cylindrical quartz rod that serves as optical window.

\section{The Instrumentation Unit}

The Instrumentation Unit is composed of one Instrumentation Base container (IB) led on the seabed and of an inductive Instrumentation Line (IL), maintained vertical by a buoy in synthetic foam, and supporting various autonomous instruments powered on internal batteries. The IU is recoverable in order to allow the change of batteries and the periodical recalibration required by some of the instruments.

The design of the IU is directly derived from the ALBATROSS project (Autonomous Line with a Broad Acoustic Transmission for Research in Oceanography and Sea Sciences) which has developed an instrumented line equipped with oceanographic sensors, that was recently deployed close to the KM3NeT-Fr site. The ALBATROSS line is totally autonomous and will communicate its data through an acoustic modem to an Instrumented Interface Module (IIM) to be connected to the KM3NeT-Fr node, as part of the Ligurian instrumentation site of the European Multidisciplinary Submarine Observatory (EMSO) network [11]. 


\subsection{The Instrumentation Base}

The instrumentation Base consists in a Titanium cylindrical container attached to a dead weight, that will contain

- an Inductive Modem (IM) that manages the inductive system. The foreseen IM system is the Seabird IMM [12], with a RS232 data interface. The IMM uses a differential phase-shift keying (DPSK) data transmission technique to achieve efficient data transmission with low error rates.

- the power system for the IMM (either provided by the CB or by an internal battery)

- the communication interface with the $\mathrm{CB}$

Based on the experience of the ALBATROSS project, the possibility to use an acoustic modem instead of an Ethernet cable is currently under study, with the potential advantage of reducing the number of wet mateable connectors and hence the risk of failure at (de)connection.

\subsection{The Instrumentation Line}

The inductive mooring line is made of a steel wire rope covered by a plastic jacket except on its ends, which are grounded in seawater, allowing a current loop through the cable. Inductive Modem devices couple to this loop inductively along the insulated part of the cable, without direct electrical connection. The signal induced on the line by the instruments is retrieved by a dedicated Seabird inductive cable coupler (ICC) [12]. The ICC is an electrical device analogous to an antenna, which inductively couples the DPSK transceiver of the IMM to the mooring line. Since there is only one current path, the instruments can only transmit data one at the time (half-duplex). The instruments will be polled periodically and sequentially using an on-shore slow control process, transiting through the CLB in the CB.

The configuration of the IL will allow the instrumentation of the full water column of the $\mathrm{KM} 3 \mathrm{NeT}$ detector. It will comprise clusters of identical instruments, distributed at different depths depending on the detector configuration (ORCA or ARCA):

- one CTD (Conductivity, Temperature, Depth) probe. These measurements are used to calculate the sound velocity as a function of water temperature, pressure and salinity according to the seawater equation of state [13]. The foreseen instrument is the SeaBird SBE 371IMP-0D0-1b model with pump and oxygen sensor, and pressure option. Even if not required for KM3NeT, the $\mathrm{O}_{2}$ measurement is of interest for the sea sciences without a large extra cost. The instrument has to be recalibrated every 2 years.

- one sound velocimeter which performs direct measurements of the celerity by measuring the time of flight of an acoustic signal over a known fixed distance. The foreseen velocimeter is the Valeport Midas Mini SVS [14]. This instrument has no native inductive interface; it will be powered and interfaced to the mooring line via the RS232-inductive coupler SBE44 from Seabird [12].

- one Acoustic Doppler Current Profiler (ADCP) that measures the current profile over a water column whose length depends on the acoustic signal frequency (from $\sim 20 \mathrm{~m}$ at 1000 $\mathrm{kHz}$ to more than $500 \mathrm{~m}$ at $75 \mathrm{kHz}$ ). This characteristic strongly influences the cost, weight and dimensions of the instrument and different options are still under study. For the dense detector configuration (ORCA), a compromise could consist in replacing one of the ADCPs by a simple, compact, single-point current meter.

The measurement periodicity of all these instruments is typically of 20 minutes. 


\section{Deployment, calibration and maintenance of the Calibration Units}

Specific mechanical tools must be designed for the KM3NeT CUs, in particular to ensure safe deployment and recovery of the IU. Base on the ALBATROSS experience, it is foreseen that the IU will be laid on the seabed from the surface boat using the winch. The instruments will be attached on the mooring line one by one on the boat while the line will descend. The deadweight of the IU will support acoustic releases that will be activated in order to recover the IL.

The IU instruments are in principle provided already calibrated. The foreseen periodicity for recalibration is every one to three years, to be adjusted taking into account the necessity of battery replacement, which will depend on the batteries capacity and on the measurement periodicity. The IL recovery will be planned during one of the sea operations for DU deployment in order to minimize the costs. It is foreseen that the first CU will be deployed on the KM3NeT-Fr site by 2017, to allow a validation of the calibration procedure with the first lines of the ORCA demonstrator array.

\section{References}

[1] KM3NeT Technical Design Report ISBN-978-90-6488-033-9

[2] R. Bruijn and D. Van Eijk [KM3NeT Collaboration], The KM3NeT multi-PMT Digital Optical Module, these proceedings, ID 1157 (2015).

[3] P. Kooijman [KM3NeT Collaboration], The Mechanical structure and deployment procedure of the KM3NeT detection unit, these proceedings, ID 1173 (2015).

[4] S. Viola [KM3NeT Collaboration], Acoustic positioning system for KM3NeT, these proceedings, ID 1169 (2015).

[5] M. Bouwhuis [KM3NeT Collaboration], Time synchronization and time calibration in KM3NeT, these proceedings, ID 1170 (2015).

[6] C. Tamburini et al. [ANTARES Collaboration], Deep-Sea Bioluminescence Blooms after Dense Water Formation at the Ocean Surface, PLOS ONE 8 (2013) e67523; H. van Haren et al. [ANTARES Collaboration], High-frequency internal wave motions at the ANTARES site in the deep Western Mediterranean, Ocean Dynamics 64 (2014)507Đ517H

[7] S. Biagi and A. Orzelli [KM3NeT Collaboration], The Central Logic Board and its auxiliary boards for the optical module of the KM3NeT detector, JINST 9 (2014) C12033.

[8] Teem Photonics website, http://www.teemphotonics.com .

[9] Photon Lines website, http://www.photonlines.fr .

[10] Newport website, http://www.newport.com .

[11] website of the EMSO Ligurian site:

http://www.emso-eu.org/infrastructure/emso-sites-description.html?id_site=9

[12] Seabird website, http://www.seabird.com/

[13] C.-T. Chen and F. J. Millero, Equation of state of seawater determined from sound speed, J. Marine Res. 36 (1978), 4.

[14] Valeport website, http://www.valeport.co.uk/ . 